\title{
Attentional Shifting and Disengagement in Rett Syndrome
}

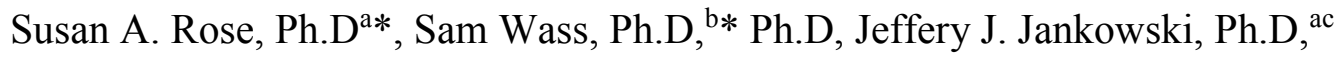
Judith F. Feldman, Ph.D, ${ }^{\mathrm{a}}$ and Aleksandra Djukic, $\mathrm{MD},{ }^{\mathrm{da}}{ }^{\mathrm{a}}$

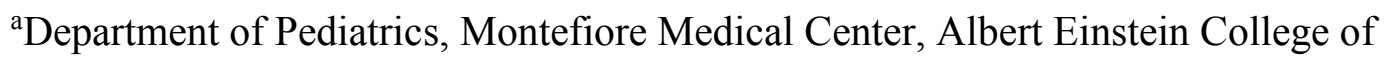
Medicine/Children's Hospital at Montefiore, Bronx, NY

${ }^{b}$ Department of Psychology, University of East London, London, England cDepartment of Social Sciences, Queensborough Community College/CUNY, United States

${ }^{\mathrm{d}}$ Rett Syndrome Center, Department of Neurology, Montefiore Medical Center, Albert Einstein College of Medicine/Children's Hospital at Montefiore, Bronx, NY

Corresponding author: Susan A. Rose, Departments of Pediatrics, Van Etten Building, Albert Einstein College of Medicine/Children's Hospital at Montefiore, 1300 Morris Park Avenue, Bronx, NY 10461. Tel: 718-839-7230. email: susan.rose@einstein.yu.edu

AUTHOR NOTE. Dr. Feldman, who made major contributions to the conceptualization, data analysis, and preliminary write-up of this work, passed away. 


\begin{abstract}
Objective. The purpose of the present study was to deepen our understanding of attention (a core cognitive ability) in Rett syndrome (RTT), an x-linked neurodevelopmental disorder caused by mutations in the MECP2 gene. We focused on two key aspects of visual orienting--shifting and disengaging attention--both of which are critical for exploring the visual world. We used gaze-based measures and eye-tracking technology to minimize demands on the limited verbal and motor abilities associated with RTT.
\end{abstract}

Method. Shifting and disengaging attention were examined in 31 children (2-12 years) with Rett Syndrome (RTT) and 31 age-matched typically-developing (TD) controls. Using the gap-overlap paradigm, the frequency/speed of shifting attention from a central to peripheral target were compared on Baseline trials, where the central stimulus disappears as the peripheral target appears, and Overlap trials, where the central stimulus remains, thus requiring disengagement. Results. Our findings revealed that children with RTT had more 'sticky fixations' $(\mathrm{p}<.001)$. That is, they had fewer saccades to the peripheral target than TD children, and this was true on both baseline (77\% vs $95 \%)$, and overlap trials ( $63 \%$ vs $90 \%)$; the younger ones also had slower saccadic reaction times $(\mathrm{SRTs})(\mathrm{p}=.04)$. Within the RTT group, SRTs correlated with symptom severity. Surprisingly, disengagement cost (the relative difference between gap and overlap SRTs) did not differ across groups. Conclusion. Our results suggest that children with Rett have difficulty shifting attention and, to a lesser extent, disengaging attention, whereas with other disorders, problems with disengagement are paramount. 
Public Statement. Rett Syndrome is a neurodevelopmental disorder characterized by profound impairments in expressive language and purposeful hand use. Given these problems, we are using eye-tracking technology to explore basic cognitive abilities. Children with Rett Syndrome had difficulty shifting gaze from a central target to a newlypresented peripheral target, whether the central one remained (competing for attention), or not. Our results suggest that children with Rett have difficulty shifting attention and, to a lesser extent, disengaging attention, whereas with other disorders, problems with disengagement are paramount.

Keywords: Rett syndrome; gap-overlap task; shifting attention; disengaging attention; eye-tracking 


\section{Attentional Shifting and Disengagement in Rett Syndrome}

Rett syndrome (RTT), an x-linked neurodevelopmental disorder affecting approximately $1 / 10,000$ females, is caused by spontaneous mutations in the $M E C P 2$ gene (Amir et al., 1999). The MECP2 gene, located on the long arm of the $\mathrm{X}$ chromosome $\mathrm{Xq} 28$, encodes methyl-CpG-binding protein $2(\mathrm{MeCP} 2)$, which is involved in regulating the transcription of other genes, as well as synaptic development and maintenance (Kaufmann, Johnston, \& Blue, 2005). RTT is characterized by apparently normal development until 6 to 18 months of age and then a severe regression that is characterized by profound impairments in expressive language and purposeful hand use (Neul et al., 2010) . In addition to these losses, the distinctive clinical features of RTT include the development of apraxia, spasticity and scoliosis, breathing irregularities (hyperventilation, breath holding, apnea), seizures, hand stereotypies, and impaired gait.

Assessments of cognitive functioning have been extremely difficult because patients with RTT are largely nonverbal and have little or no purposeful hand use. These profound impairments make standard neuropsychological testing impossible, leaving the cognitive phenotype of RTT largely a mystery. However, recent work using eye-tracking, which minimizes the impact of these motoric and language problems, shows that communicative eye gaze is relatively preserved in RTT (Ahonniska-Assa et al., 2018; Baptista, Mercadante, Macedo, \& Schwartzman, 2006; Djukic \& Valicenti McDermott, 
2012; Djukic, Valicenti McDermott, Mavrommatis, \& Martins, 2012 ; Schwartzman, Velloso, D'Antino, \& Santos, 2015). Eye tracking technology thus holds promise for unlocking the cognitive world of children with RTT. It offers a way of assessing the cognitive abilities of these children - illuminating capabilities that are relatively spared, providing an understanding of the developmental trajectories, and identifying areas of relative weakness.

In our own lab, we initially showed that eye-tracking technology was indeed usable with the RTT population and established that children with RTT showed selective attention to salient areas and novel elements (Djukic \& Valicenti McDermott, 2012; Djukic et al., 2012 ). We then began to utilize this technique for systematic studies examining memory and attention. The memory studies used the visual pairedcomparison paradigm, in which two identical stimuli are presented side by side for familiarization (10 seconds for Faces; 5 seconds for Patterns) and then, in an immediate test, the familiar and a new target are paired. Although children with RTT recognized simple patterns and faces (novelty scores $>50 \%$ ), they did not do so as well as did their typically developing age-matched controls (Djukic, Rose, Jankowski, \& Feldman, 2014; Rose et al., 2013; Rose, Djukic, Jankowski, Feldman, \& Rimler, 2016). Of particular importance were findings suggesting that these differences in memory were coupled with problems in attention.

These attentional problems are of particular concern, since attention is a foundational ability closely linked to cognitive development. Indeed, attention has been 
found to underpin the development of a wide range of abilities in typically developing children, and other risk groups. For example, infant and toddler look duration and shift rate were related to later IQ, language, and executive functioning (Rose, Feldman, \& Jankowski, 2015; Rose, Feldman, \& Jankowski, 2012; Rose, Feldman, Jankowski, \& Van Rossem, 2008); habituation of attention were related to later IQ and academic achievement (Bornstein, Hahn, \& Wolke, 2013); and attentional control and visual search were related to later receptive language (Whedon, Perry, Calkins, \& Bell, 2016).

While attention is multi-faceted, subserved by distinct neural systems (Fan, McCandliss, Fossella, Flombaum, \& Posner, 2005; Posner \& Petersen, 1990), one of the core components is orienting, which involves attentional shifting and disengagement. To visually explore the world, one must be able to flexibly break attention, or disengage, in order to re-orient elsewhere. Attentional shifting is thought to involve largely low level oculomotor networks, especially pathways from the retina to the superior colliculus (Karatekin, Marcus, \& White, 2007), whereas attentional disengagement is thought to rely more heavily on prefrontal and parietal areas of the cortex (Csibra, Johnson, \& Tucker, 1997; Csibra, Tucker, \& Johnson, 1998).

While we know of no neuropsychological studies targeting oculomotor abilities in children with RTT, or studies targeting the orienting network, the attentional atypicalities we found previously suggest that they may have difficulty disengaging attention ('sticky fixations') and moving their gaze throughout the visual world. Sticky fixations severely limit the information taken in from the visual world and thus slow the development of 
other cognitive abilities. Studies of children with other disorders have identified atypicalities in disengaging attention; such disorders include autism (Landry \& Bryson, 2004; Sabatos-DeVito, Schipul, Bulluck, Belger, \& Baranek, 2016; dis et al., 2005), highrisk infants subsequently diagnosed with autism (Bedford et al., 2014; Elsabbagh et al., 2013; Elsabbagh et al., 2009 ), and children with attention-deficit hyperactivity disorder (ADHD) (Cairney et al., 2001). While children with these disorders have difficulty turning to a target when they must first disengage from an existing point of focus, they have little difficulty doing so if the target disappears. These abilities have not, to our knowledge, been examined in children with RTT.

In the present study, we used a 'gap-overlap task' to determine whether children with RTT have difficulty shifting and/or disengaging attention. Children were tested with a computerized version (S. Wass, Porayska-Pomsta, \& Johnson, 2011) of the task used by Elsabbagh (Elsabbagh et al., 2009). In this task, a central fixation stimulus appeared first and then, after a brief delay, a peripheral target appeared. On Baseline Trials, the central stimulus disappeared when the peripheral target appeared; on Overlap Trials, the central stimulus remained present throughout the trial (and competed for attention). The ability to shift and disengage were assessed by considering both the frequency of saccades to the peripheral target and saccadic reaction time (SRT).

It was hypothesized that children with RTT would have more difficulty in disengaging attention than the TD group, and that this would be evidenced by (1) fewer saccades to the peripheral target on overlap trials, and (2) slower saccadic reaction time 
(SRT) on these trials as well. We also examined how two aspects of data quality (precision and robustness; (S.V. Wass, Forssman, \& Leppanen, 2014) impacted group differences, and how symptom severity related to performance in children with RTT.

\section{Method}

The present study was part of a larger project aimed at identifying the nature of attentional impairments in children with RTT. The participants in the Gap-Overlap task discussed here also received two other tasks, one assessing sustained attention (Rose, Wass, Jankowski, Feldman, \& Djukic, 2017), and another assessing selective attention (submitted). Each task took about 2-3 minutes; blocks of trials from the three tasks were interleaved, with the entire session lasting approximately 10 minutes. All tasks were gaze-contingent and utilized the same eye-tracking apparatus.

\section{Participants}

Participants were 31 girls who had been clinically diagnosed as having classical RTT (Neul et al., 2010), consecutively recruited from the Rett Center at the Children's Hospital of Montefiore $(M=7.33$ years; $S D=3.07$, range=2-12; $\mathrm{Cl}[6.2,8.4])$ and a comparison group of 31 typically developing (TD) girls ( $M=7.34$ years; $S D=2.87$, range $=2-12 ; \mathrm{Cl}[6.3,8.4])$; the groups did not differ in chronological age, $t(60)=.09$, ns. (It should be noted that the severe limitations suffered by children with RTT in verbal ability, and purposeful hand movements, preclude the neuropsychological testing that would be needed for matching groups on mental age.) 
The children in the TD group, recruited from Outpatient Clinics of the same hospital, were family members of patients visiting pediatric specialty clinics. The TD group was screened to exclude any children with significant neurological or chromosomal disorders, sensory impairment, neurodevelopmental disorders, or firstdegree relatives with neurodevelopmental disorders.

Children were excluded from the final sample if they could not successfully complete the calibration procedure $(N=3 \mathrm{RTT})$, were too overactive/restless to complete the procedure ( $N=5 \mathrm{RTT})$, had fewer than 12 responses in either baseline or overlap condition ( $N=3$ RTT, $N=1 \mathrm{TD}$ ), or had compromised data due to technical difficulties $(N=1 \mathrm{TD})$. The children excluded did not differ in clinical/background factors from the rest of the sample.

\section{Clinical characteristics of the RTT sample}

Table 1 shows, for each child in the RTT group, their genetic mutation, age at test, age at regression (all had completed active regression and were in Stage 3, a period of stabilization or plateau), score on the Rett Syndrome Severity Scale (RSSS)(Kaufmann et al., 2012). The RSSS, a clinician-rated scale that represents an aggregate measure of the severity of clinical symptoms, includes seven subscales: seizure frequency/manageability, respiratory irregularities, scoliosis, ability to walk, hand use, speech, and sleep problems. Each parameter is scored on a 4-point Likert scale from 0 (absent/normal) to 3 (severe). Composite scores on the RSSS, created by taking the mean of all subscales, averaged $7.97(S D=2.37$; range $=4-14 ; 95 \%$ confidence interval $(\mathrm{CI})$ 
$[7.1,8.4]$ ). Of the RTT participants, $45.2 \%$ were ambulatory (able to walk unaided or with support) and $38.7 \%$ had a history of seizures. Additionally, it should be noted that the two age groupings of RTT children created by a median split (described below under data analysis) did not significantly differ from one another either in age at regression $t(29)=1.41, p=.17$ or RSSS $t(29)=1.14, p=.26$. The protocol was approved by the institutional review board (IRB Protocol \#3203) and written consent was obtained for all participants.

Table 1 goes about here

\section{Apparatus}

The stimuli were presented on a 23-inch flat panel monitor (resolution, $1024 \times 768$ pixels), and eye-gaze recorded using a Tobii X2-60 infrared eyetracker (Tobii Technologies, Dandyred, Sweden). The computer was programmed using Matlab and Psychtoolbox, with Talk2Tobii software used to allow for a live gaze-contingent interface via Matlab during stimulus presentation. Manufacturer-supplied algorithms for pupil, corneal reflection, and face identification were used during eye-tracking; gaze data were sampled at $60 \mathrm{~Hz}$. The xy-coordinates of the left and right eye gaze positions were recorded separately, and then, to improve the quality of the data, averaged for analyses. In the rare case where one of the xy-coordinates for one of the eyes was invalid, only the eye with valid xy-coordinates was used. 


\section{Procedure}

Children were tested in a quiet, dimly lit room, seated approximately $45 \mathrm{~cm}$ from the monitor. To minimize body and head movements, all children in the RTT group (and all TD participants $<5$ years) were seated on a caregiver's lap. Caregivers kept their eyes closed during testing.

\section{Calibration}

At the start of the session, children completed a 5-point calibration procedure, in which pulsing colored blocks $\left(1^{\circ}\right.$ to $\left.1.5^{\circ}\right)$ appeared in random succession at the center and corners of the screen. If the fixation points did not cluster, or any targets were missed, the calibration was repeated until a satisfactory calibration was achieved. Each calibration attempt took less than a minute.

\section{Gap-Overlap Task: Design and Stimuli}

We measured attentional disengagement with a gap-overlap paradigm. Children were instructed to 'look at the TV.' All trials began with a $500 \mathrm{~ms}$ presentation of a blank white screen, followed by the appearance of a colorful, animated, rotating clock face in the center of the screen $\left(4.5^{\circ}\right)$. As soon as the child fixated the central stimulus, a pulsating cartoon cloud $\left(3^{\circ}\right)$ appeared $20^{\circ}$ to the left or right of the central stimulus. As soon as the child looked to the cloud, it disappeared and was replaced by a cartoon picture that performed a brief animation before disappearing. If the central stimulus was not fixated within $5000 \mathrm{~ms}$, the lateral target was presented anyway, and remained until fixated, or for $4000 \mathrm{~ms}$. (Decisions to proceed to the next stimulus were automated and 
based on the real-time eye-tracker data.) On Baseline Trials, the central target disappeared when the peripheral target was presented; on Overlap Trials the central target remained during the presentation of the lateral target. Lateral targets appeared equally often, and unpredictably, to the left and right of center. There were two blocks of 12 trials (with the order of the 6 baseline trials and 6 overlap trials within each block randomized), for a total of 24 trials. The two blocks of trials, each lasting less than 2 minutes, were interleaved with the two other attention tasks.

\section{Data Analyses}

For trials to be scorable, the child had to fixate the center target at the start of the trial. Although the TD group had more scorable trials than the RTT group, $99 \%$ vs $89 \%$, $t(60)=3.80, p=.001$, both percentages were high, indicating that the task effectively captured the attention and interest of children in both groups.

Two measures of performance were analyzed: frequency and latency to look to the peripheral target, with the latter indexed by saccadic reaction time (SRT). SRTs in baseline and overlap conditions were measured as the time elapsed between the appearance of the peripheral target and the reported position of gaze leaving the central area (a $9^{\circ}$ box around the central target). For an SRT to be considered valid the child had to: (a) look to the central stimulus location; and (b) make an eye movement to the peripheral target within $4000 \mathrm{~ms}$ of its onset. If there was no shift in gaze toward the peripheral target within this period, no SRT was recorded and the trial was considered a failure to disengage. Also, to obtain some idea of developmental change, we compared 
the younger vs older children by using a median split for both groups, $<8$ years vs $\geq 8$ years, the same cut-point that was used previously with this cohort (Rose et al., 2017).

All measures were examined for normality and outliers and analyzed using ANOVA. Because their distributions were skewed, for statistical analysis, SRT latencies were $\log$ transformed. (Raw scores are reported in tables and figures for ease of understanding.) All effects were evaluated at a .05 level of significance; SPSS (version 24) was used in all analyses; Bonferroni-adjusted significance tests were used for all pairwise comparisons. Effect sizes for tests of differences are reported as partial etasquared; effect sizes for tests of association are reported as Pearson's $r$.

\section{Quality of eye-tracking data}

Recent work by Wass and colleagues suggests that two aspects of the eyetracking data, precision and robustness, could influence the recordings of fixation duration, and should be considered when examining group differences in outcome measures (S.V. Wass et al., 2014).

Precision. Low precision, which occurs when there is a difference between the reported position of gaze of the pupil and the 2-D projection of the target, happens when one of the elements (pupil, corneal reflection, head position) is incorrectly identified by the eye-tracking software. To calculate precision, we: (1) exported the raw sample-bysample $\mathrm{x}$ and $\mathrm{y}$ coordinates obtained during tracking into Matlab; (2) smoothed the data using a down-sampling procedure that chunked the data into consecutive $100 \mathrm{~ms}$ windows, and then calculated a single median for each window; (3) compared the 
average difference between the down-sampled and unfiltered data across all windows. These calculations were done separately for the $\mathrm{x}$ and $\mathrm{y}$ coordinates and then the final medians were averaged. Less precise tracking is indexed by a higher value of the metric (i.e., greater discrepancy between the down-sampled and unfiltered data).

Robustness. Low robustness occurs when the tracker fails to report on position of gaze at all, leading the data to 'flicker' off for periods (ranging from a few ms to several seconds). Longer periods of missing data may be caused by the child not looking at the screen; shorter periods are caused by the eye-tracker failing to detect the corneal reflection and pupil consistently and reliably (e.g., the corneal reflection is obscured by an eyelid, common in children who fidget/move). To calculate robustness, we: (1) imported raw data from the recording software to Matlab, (2) performed linear interpolation on gaps $\leq 150 \mathrm{~ms}$ (since these very short periods of missing data were considered too brief to contain a complete saccade-fixation-saccade sequence, and thus were unlikely to have affected our results (Wass, Forssmann \& Leppanen, 2014), 3) calculated the mean duration of all remaining usable data fragments. Less robust data is indexed by a lower value of the metric (i.e., shorter average fragment durations, due to more flicker).

We examined group differences in precision and robustness using 2 (Group: RTT vs TD) x 2 (Age: younger vs older) ANOVAs. Although there were no significant effects for precision, $F(1,61)=.01, p<.93, \eta_{\mathrm{p}}{ }^{2}=.00$, there was a significant Group effect for robustness, $F(1,61)=5.74, p=.04, \eta_{\mathrm{p}}{ }^{2}=.09$, due to the average fragment duration being 
shorter for the RTT group $(M=1.60 \mathrm{~s}, S D=.88$, CI $[1.27,1.92])$ than the TD group $(M=$ $3.07 \mathrm{~s}, S D=4.30$, CI $[1.49,4.64])$. Thus, the RTT group had more periods of missing or flickering data than the TD group.

In order to be able to determine whether robustness affected group difference on the gap-overlap tasks, we divided the sample into two, using a median split that was based on the entire sample, and entered robustness as a dichotomous factor in the ANOVAs. Thus, there was a subgroup lower on robustness ( $N=20$ RTT and $12 \mathrm{TD})$, and one higher on this factor $(N=13$ RTT and 19 TD).

\section{Results}

\section{Frequency of Gaze Shifts}

The percentages of baseline and overlap trials where the child looked to the peripheral target are shown in Fig 1. These data were analyzed in a 2 (Group: RTT vs TD) x 2 (Age: younger vs older) x 2 (Robustness: lower vs higher) x 2 (Condition: baseline vs overlap) ANOVA, with repeated measures on the last factor. There was a significant effect for Group, $F(1,54)=20.25, p<.001, \eta_{\mathrm{p}}{ }^{2}=.273$, indicating that children with RTT were less likely to orient to the peripheral target than their TD counterparts, and a significant effect for Condition, $F(1,58)=23.23, p<.001, \eta_{\mathrm{p}}{ }^{2}=.27$, indicating that children in both groups showed significantly fewer saccades to the peripheral target in the overlap condition, where disengagement was required, than in the baseline condition. There was also a significant effect for robustness, $F(1,54)=4.17, p=.04, \eta_{\mathrm{p}}{ }^{2}=.072$, with more robust data (less flicker) associated with more frequent orienting to the peripheral 
target. However, the robustness $\mathrm{x}$ group interaction was not significant, $F(1,54)=.74, p=$ .40 , indicating that flicker did not account for group difference in the frequency of orienting to the peripheral target. Overall, the children with RTT shifted their gaze to the peripheral target on $77 \%$ of the baseline trials $(\mathrm{SD}=20.88,(\mathrm{CI})[69,85$, ] and $63 \%$ of the overlap trials ( $\mathrm{SD}=24.22$, CI $[54,72]$.) whereas the corresponding figures for their TD counterparts were $95 \%(\mathrm{SD}=9.58,(\mathrm{CI})[92,99]$,$) and 90 \%(\mathrm{SD}=16.76,(\mathrm{CI})[84,96])$.

Fig 1 goes about here.

\section{Latency of Gaze Shifts (Saccadic Reaction Times)}

The SRTs shown in Fig 2 are based on trials where the children initiated a shift in gaze from the center to the peripheral target. These results were analyzed using a 2 (Group: RTT vs TD) x 2 (Age: younger vs older) x 2 (Robustness: lower vs higher) x 2 (Condition: baseline vs overlap) ANOVA, with repeated measures on condition. There was a significant main effect for Condition, $F(1,54)=15.85, p<.001, \eta_{\mathrm{p}}{ }^{2}=.227$, reflecting faster SRTs on baseline than overlap trials, and a significant Group x Age interaction, $F(1,54)=4.25, p=.04, \eta_{\mathrm{p}}{ }^{2}=.073$, indicating that the younger children with RTT had longer latencies than their TD counterparts. Robustness had no significant effect on latency scores, $F(1,54)=2.05, p=.16, \eta_{\mathrm{p}}{ }^{2}=.037$.

It should be noted that 'disengagement cost,' the difference between SRT on baseline and overlap trials, also did not differ for the two groups, as indicated by the non- 
significant group $\mathrm{x}$ condition interaction, $\left.F(1,54)=.21, p=.65, \eta_{\mathrm{p}}{ }^{2}=.004\right)$. Thus, while both groups took significantly longer to saccade to the peripheral target in the overlap than baseline condition, children with RTT did not take proportionally longer than their TD counterparts.

Fig 2 goes about here.

\section{Correlations of Performance to Clinical Severity of RTT Symptomatology}

We next examined whether the clinical severity of RTT (as reflected in higher RSSS scores) or age-at-regression related to the major measures of performance:

frequency of gaze shifts and SRTs on both baseline and overlap trials, and disengagement cost (the difference between SRT on baseline and overlap trials). We used one-tailed tests since we expected that earlier regression and more severe symptomatology would be related to fewer gaze shifts, longer SRTs, and a greater disengagement cost.

The results indicated that more severe symptomatology (a higher RSSS composite score) was associated with longer SRTs on baseline trials $(r=.30, p=.05)$ and greater disengagement $\operatorname{cost}(r=.32, p=.04)$. Earlier onset of symptomatology (earlier regression) was associated with longer SRTs on overlap trials $(r=.37, p=.02)$. None of the other correlations were significant ( $r$ r ranged from -.15 to .19).

\section{Discussion}


Our understanding of the cognitive phenotype of RTT remains rudimentary, impeded by the inability of girls with this disorder to speak or use their hands purposely. Eye tracking has begun to be used to overcome this problem (Djukic et al., 2016; Djukic et al., 2014; Rose et al., 2013; Rose et al., 2016; Rose et al., 2017) and, in the present study, we used this technology to focus on attention shifting and disengagement. To do this, we used a gap-overlap task, which contrasts the ability to shift from a central to a peripheral target in two conditions: (a) where the central target disappears as the peripheral one appears (baseline), and (b) where the central target remains present during the appearance of the peripheral one (overlap), and thus competes for attention.

Children with RTT had considerably more difficulty shifting attention than the TD group, as indicated by lower accuracy scores in both the overlap (63\% vs $90 \%)$ and baseline conditions (77\% vs 95\%). While the performance of children with RTT (like that of the TD group) was better on baseline trials, their performance in both conditions was relatively poor. In short, the children with RTT showed more 'sticky fixations' than the TD group, turning to the peripheral target less frequently in both conditions.

When they did fixate on the peripheral target, saccade latencies were longer for the younger children with RTT than for their TD counterparts. This difference between groups was no longer evident at the older age, due largely to age-related improvement (decreased latencies) in the RTT group. These results are in agreement with a large literature showing that saccade -latency is longer in children than in adults and that it shortens progressively over childhood (Ross, Radant, \& Hommer, 1994). Since this 
study was not a longitudinal study, and the sample size was small, further study is needed to know whether we are witnessing a similar developmental phenomenon in RTT. Neither the severity of RTT nor the age at regression would appear to account for developmental improvement, since the age groups did not differ on either factor. Surprisingly however, the baseline-overlap difference in SRTs was comparable across group and age. Thus, while the children with RTT were less likely to shift their attention and gaze to the peripheral target, and somewhat slower when they did, the 'disengagement cost' (the relative difference between gap and overlap SRTs) was similar.

While the gap-overlap task has been used with a number of risk groups, the findings associated with RTT appear to be disorder specific in two respects. First, children with RTT showed a markedly reduced tendency to turn to the peripheral target not only on overlap trials, but even in the baseline condition. While we had hypothesized that they would have difficulty on overlap trials, their difficulty on baseline trials was unexpected. With other groups, such failures tend to be rare, and when they do occur, are largely restricted to overlap trials, where there is competition between the central and peripheral targets (Elsabbagh et al., 2013; Elsabbagh et al., 2009; Hunnius, Geuze, Zweens, \& Bos, 2008; Sabatos-DeVito et al., 2016). Second, children with RTT did not show any greater disengagement cost than the TD group (i.e., the longer latencies on overlap trials were similar across age and group). By contrast, children with other developmental disorders often show markedly longer latencies on overlap trials than their typically developing counterparts, resulting in a markedly greater disengagement cost 
(Elsabbagh et al., 2013; Elsabbagh et al., 2009; Hunnius et al., 2008; Landry \& Bryson, 2004; Zwaigenbaum et al., 2005). .

Our results are consistent with there being a deficit in shifting attention in Rett but not specifically with a deficit in disengaging attention on top of that. This deficit is likely to involve difficulty in oculo-motor processes. Oculomotor processes, which underlie the control of planned eye movements, are thought to be involved in mediating baseline performance. These processes involve pathways from the retina to the superior colliculus (Hood \& Atkinson, 1993), or those needed for cortical disinhibition of the superior colliculus (Csibra et al., 1997; Csibra et al., 1998). Performance on overlap trials, where disengagement is required, is thought to also recruit areas of the parietal cortex, as indicated by prolonged ERP activity observed in this region prior to saccade execution (Csibra et al., 1997; Csibra et al., 1998).

There are two further aspects of the study that warrant comment. First, it should be noted that this is the first study to examine the quality of eye tracking data in RTT. Two major aspects were quantified: precision and robustness. The precision of the recordings did not differ between groups, indicating that the eye tracker picked up the location of looks as well for children with RTT as for TD children. However, the robustness of the data, as reflected in the amount of 'flicker' or missing segments in the data, was poorer in RTT. The increased flicker is likely caused by the difficulty these children often have in controlling their head movement, which could mean that the head is more likely to be positioned at an oblique angle to the screen, meaning that, for 
example, the corneal reflection is more likely to be obscured by eyelid, making tracking unreliable (S.V. Wass, 2015). However, it should be pointed out that robustness did not account for any of the differences found between groups in either the frequency or latency of SRTs.

Second, it should be noted that, within the RTT group, some aspects of performance were related to symptomatology. In particular, slower SRTs, and higher disengagement costs, were related to more severe symptomatology (as assessed by the RSSS) and/or earlier regression. These relations raise the possibility that measures of disengagement and SRT may prove to be useful biomarkers for assessing the effectiveness of therapeutic interventions.

The current findings, in conjunction with earlier work, provide growing evidence of aberrant attention in RTT. Earlier studies identified limitations in sustained attention, distractibility, and the ability to anticipate predictable events (Rose et al., 2016; Rose et al., 2017). Here, we show that children with RTT also have problems shifting attention, a key aspect of attentional control and flexibility. Some of these difficulties are shared with those found in children with other neurodevelopmental disorders and those with perinatal brain injury, including focal lesions (Mercuri et al., 1996), hypoxic-ischaemic encephalopathy (Mercuri et al., 1997), perinatal injury of white matter due to prematurity (Atkinson et al., 2008), Williams syndrome (Atkinson et al., 2003), and risk for autism (Elsabbagh et al., 2013). Further research is needed to more fully establish the cognitive profile uniquely associated with RTT. Attentional flexibility and gaze shifting are 
important for exploring the world and regulating looking during social interaction. Thus, problems in flexibly switching attention are likely to have widespread ramifications for cognitive growth, particularly for the development and execution of more complex cognitive abilities in this population. 


\section{References}

Ahonniska-Assa, J., Polack, O., Saraf, E., Wine, J., Silberg, T., Nissenkorn, A., \& BenZeev, B. (2018). Assessing cognitive functioning in females with Rett syndrome by eye-tracking methodology. European Journal of Paediatric Neurology, 22, 3945.

Amir, R. E., Van den Veyver, I. B., Wan, M., Tran, C. Q., Francke, U., \& Zoghbi, H. Y. (1999). Rett syndrome is caused by mutations in X-linked MECP2, encoding methyl-CpG-binding protein 2. Nature Genetics,, 23(2), 185-188. doi: $10.1038 / 13810$

Atkinson, J., Braddick, O., Anker, S., Curran, W., Andrew, R., Wattam-Bell, J., \& Braddick, F. (2003). Neurobiological models of visuospatial cognition in children with Williams syndrome: measures of dorsal-stream and frontal function. Developmental Neuropsychology, 23(1-2), 139-172. doi:10.1080/87565641.2003.9651890

Atkinson, J., Braddick, O., Anker, S., Nardini, M., Birtles, D., Rutherford, M. A., . . . Cowan, F. M. (2008). Cortical vision, MRI and developmental outcome in preterm infants. Archives of Disease in Childhood. Fetal and Neonatal Edition, 93(4), F292-297. doi:10.1136/adc.2007.116988

Baptista, P. M., Mercadante, M. T., Macedo, E. C., \& Schwartzman, J. S. (2006). Cognitive performance in Rett syndrome girls: a pilot study using eyetracking 
technology. Journal of Intellectual Disabilities Research, 50(Pt 9), 662-666. doi:10.1111/j.1365-2788.2006.00818.x

Bedford, R., Pickles, A., Gliga, T., Elsabbagh, M., Charman, T., Johnson, M. H., \& Team, Basis. (2014). Additive effects of social and non-social attention during infancy relate to later autism spectrum disorder. Developmental Science, 17(4), 612-620.

Bornstein, M.H., Hahn, C.S., \& Wolke, D. (2013). Systems and cascades in cognitive development and academic achievement. Child Development, 84(1), 154-162. doi:10.1111/j.1467-8624.2012.01849.x

Cairney, S., Maruff, P., Vance, A., Barnett, R., Luk, E., \& Currie, J. (2001). Contextual abnormalities of saccadic inhibition in children with attention deficit hyperactivity disorder. Experimental Brain Research, 141(4), 507-518.

doi:10.1007/s002210100890

Csibra, G., Johnson, M. H., \& Tucker, L. A. (1997). Attention and oculomotor control: a high-density ERP study of the gap effect. Neuropsychologia, 35(6), 855-865.

Csibra, G., Tucker, L. A., \& Johnson, M. H. (1998). Neural correlates of saccade planning in infants: a high-density ERP study. International Journal of Psychophysiology, 29(2), 201-215.

Djukic, A., Holtzer, R., Shinnar, S., Muzumdar, H., Rose, S. A., Mowrey, W., ... Moshe, S. L. (2016). Pharmacologic treatment of Rett Syndrome with glatiramer acetate. Pediatric Neurology, 61, 51-57. doi:10.1016/j.pediatrneurol.2016.05.010 
Djukic, A., Rose, S. A., Jankowski, J. J., \& Feldman, J. F. (2014). Rett syndrome: recognition of facial expression and its relation to scanning patterns. Pediatric Neurology, 51(5), 650-656. doi:10.1016/j.pediatrneurol.2014.07.022

Djukic, A., \& Valicenti McDermott, M. (2012). Social preferences in rett syndrome. Pediatric Neurology, 46(4), 240-242. doi:10.1016/j.pediatrneurol.2012.01.011

Djukic, A., Valicenti McDermott, M., Mavrommatis, K., \& Martins, C.L. (2012 ). Rett syndrome: Basic features of visual processing--a pilot study of eye-tracking. Pediatric Neurology, 47, 25-29.

Elsabbagh, M., Fernandes, J., Jane Webb, S., Dawson, G., Charman, T., Johnson, M. H., \& British Autism Study of Infant Siblings, Team. (2013). Disengagement of visual attention in infancy is associated with emerging autism in toddlerhood. Biological Psychiatry, 74(3), 189-194. doi:10.1016/j.biopsych.2012.11.030

Elsabbagh, M., Volein, A., Holmboe, K., Tucker, L., Csibra, G., Baron-Cohen, S., . . . Johnson, M. H. (2009). Visual orienting in the early broader autism phenotype: disengagement and facilitation. Journal of Child Psychology and Psychiatry, 50(5), 637-642. doi:10.1111/j.1469-7610.2008.02051.x

Fan, Jin, McCandliss, Bruce D., Fossella, John, Flombaum, Jonathan I., \& Posner, Michael I. (2005). The activation of attentional networks. NeuroImage, 26(2), $471-479$.

Hood, B.M., \& Atkinson, J. (1993). Disengaging visual attention in the infant and adult. Infant Behavior and Development, 16(4), 405-422. 
Hunnius, S., Geuze, R. H., Zweens, M. J., \& Bos, A. F. (2008). Effects of preterm experience on the developing visual system: a longitudinal study of shifts of attention and gaze in early infancy. Developmental Neuropsychology, 33(4), 521535. doi:10.1080/87565640802101508

Karatekin, C., Marcus, D. J., \& White, T. (2007). Oculomotor and manual indexes of incidental and intentional spatial sequence learning during middle childhood and adolescence. Journal of Experimental Child Psychology, 96(2), 107-130. doi:10.1016/j.jecp.2006.05.005

Kaufmann, W. E., Johnston, M. V., \& Blue, M. E. (2005). MeCP2 expression and function during brain development: implications for Rett syndrome's pathogenesis and clinical evolution. Brain and Development, 27 Suppl 1, S77-S87. doi:10.1016/j.braindev.2004.10.008

Kaufmann, W. E., Tierney, E., Rohde, C. A., Suarez-Pedraza, M. C., Clarke, M. A., Salorio, C. F., . . Naidu, S. (2012). Social impairments in Rett syndrome: characteristics and relationship with clinical severity. Journal of Intellectual Disabilities Research, 56(3), 233-247. doi:10.1111/j.1365-2788.2011.01404.x

Landry, R., \& Bryson, S.E. (2004). Impaired disengagement of attention in young children with autism. Journal of Child Psychology and Psychiatry, 45, 11151122. 
Mercuri, E., Atkinson, J., Braddick, O., Anker, S., Cowan, F., Rutherford, M., . . . Dubowitz, L. (1997). Visual function in full-term infants with hypoxic-ischaemic encephalopathy. Neuropediatrics, 28(3), 155-161. doi:10.1055/s-2007-973693

Mercuri, E., Atkinson, J., Braddick, O., Anker, S., Nokes, L., Cowan, F., . . Dubowitz, L. (1996). Visual function and perinatal focal cerebral infarction. Archives of Disease in Childhood. Fetal and Neonatal Edition, 75(2), F76-81.

Neul, J. L., Kaufmann, W. E., Glaze, D. G., Christodoulou, J., Clarke, A. J., BahiBuisson, N., . . Percy, A. K. (2010). Rett syndrome: revised diagnostic criteria and nomenclature. Annals of Neurology, 68(6), 944-950. doi:10.1002/ana.22124

Posner, M.I., \& Petersen, S. (1990). The attention system of the human brain. Annual Review of Neuroscience, 13, 25-42.

Rose, S. A., Djukic, A., Jankowski, J. J., Feldman, J. F., Fishman, I., \& ValicentiMcdermott, M. (2013). Rett syndrome: an eye-tracking study of attention and recognition memory. Developmental Medicine and Child Neurology, 55(4), 364371. doi:10.1111/Dmen.12085

Rose, S. A., Djukic, A., Jankowski, J. J., Feldman, J. F., \& Rimler, M. (2016). Aspects of attention in Rett Syndrome. Pediatric Neurology, 57, 22-28. doi:10.1016/j.pediatrneurol.2016.01.015

Rose, S. A., Feldman, J. F., \& Jankowski, J .J. (2015). Pathways From toddler information processing to adolescent lexical proficiency. Child Development, 86(6), 1935-1947. doi:10.1111/cdev.12415 
Rose, S. A., Feldman, J. F., \& Jankowski, J. J. (2012). Implications of infant cognition for executive functions at age 11. Psychological Science, 23(11), 1345-1355. doi:10.1177/0956797612444902

Rose, S. A., Feldman, J.F., Jankowski, J.J., \& Van Rossem, R. (2008). A cognitive cascade in infancy: Pathways from prematurity to later mental development Intelligence, 36, 367-378.

Rose, S. A., Wass, S., Jankowski, J. J., Feldman, J. F., \& Djukic, A. (2017). Sustained attention in the face of distractors: A study of children with Rett syndrome. Neuropsychology, 31(4), 403-410. doi:10.1037/neu0000369

Ross, R.G., Radant, A.D., \& Hommer, D.W. (1994). Saccadic eye movements in normal children from 8 to 15 years of age: A developmental study of visuaspatial attention. Journal of Autism and Developmental Disorders, 24, 413-431.

Sabatos-DeVito, M., Schipul, S. E., Bulluck, J. C., Belger, A., \& Baranek, G. T. (2016). Eye tracking reveals impaired attentional disengagement associated with sensory response patterns in children with autism. Journal of Autism and Developmental Disorders, 46(4), 1319-1333. doi:10.1007/s10803-015-2681-5

Schwartzman, J. S., Velloso, Rde L., D'Antino, M. E., \& Santos, S. (2015). The eyetracking of social stimuli in patients with Rett syndrome and autism spectrum disorders: a pilot study. Arquivos de Neuro-Psiquiatria, 73, 402-407.

Wass, S., Porayska-Pomsta, K., \& Johnson, M. H. (2011). Training attentional control in infancy. Current Biology, 21(18), 1543-1547. doi:10.1016/j.cub.2011.08.004 
Wass, S.V. . (2015). The uses of eye-tracking with children. In J.R. \& Prior Van Herwegen, J.J. (Ed.), Research Methods in Developmental Psychology. London: Routledge.

Wass, S.V., Forssman, L., \& Leppanen, J. M. (2014). Robustness and precision: How data quality may influence key dependent variables in infant eye-tracker analysis. Infancy, 19(5), 427-460.

Whedon, M., Perry, N. B., Calkins, S. D., \& Bell, M. A. (2016). Changes in frontal EEG coherence across infancy predict cognitive abilities at age 3: The mediating role of attentional control. Developmental Psychology. doi:10.1037/dev0000149

Zwaigenbaum, L., Bryson, S., Rogers, T., Roberts, W., Brian, J., \& Szatmari, P. (2005). Behavioral manifestations of autism in the first year of life. International Journal for Developmental Neuroscience, 23(2-3), 143-152.

doi:10.1016/j.ijdevneu.2004.05.001 


\section{Acknowledgements}

We thank the participants and their families for their cooperation and effort. This research was funded by a grant from the International Rett Syndrome Foundation (IRSF). 
Table and Figure Legends

Table 1: Clinical and genetic characteristics of the children with Rett syndrome (RTT)

Fig 1 Frequency of gaze shifts on baseline and overlap trials for typically developing children (TD) and those with Rett Syndrome (RTT).

Fig 2: Saccadic reaction times on baseline and overlap trials for typically developing children (TD) and those with Rett Syndrome (RTT). 
Table 1: Clinical and genetic characteristics of the children with Rett syndrome

\begin{tabular}{|c|c|c|c|c|}
\hline Patient & Genetics & $\begin{array}{l}\text { Age at Testing } \\
\text { (years) }\end{array}$ & $\begin{array}{l}\text { Age at Regression } \\
\text { (months) }\end{array}$ & $\begin{array}{c}\text { RSSS } \\
\text { Total Score }\end{array}$ \\
\hline 1 & $\mathrm{R} 133 \mathrm{C}$ & 8 & 15 & 8 \\
\hline 2 & R306C & 11 & 18 & 5 \\
\hline 3 & R133C & 7 & 15 & 7 \\
\hline 4 & Deletion & 6 & 12 & 9 \\
\hline 5 & R270X & 5 & 30 & 5 \\
\hline 6 & deletion & 11 & 27 & 8 \\
\hline 7 & Deletion & 9 & 18 & 6 \\
\hline 8 & R168X & 7 & 15 & 9 \\
\hline 9 & $\mathrm{R} 255 \mathrm{X}$ & 10 & 12 & 14 \\
\hline 10 & C916T & 4 & 24 & 7 \\
\hline 11 & R168X & 4 & 15 & 7 \\
\hline 12 & Deletion & 11 & 18 & 6 \\
\hline 13 & T158M & 12 & 12 & 11 \\
\hline 14 & T158M & 4 & 13 & 4 \\
\hline 15 & R168X & 9 & 36 & 10 \\
\hline 16 & R294X & 5 & 17 & 7 \\
\hline 17 & R168X & 4 & 32 & 11 \\
\hline 18 & R168X & 2 & 6 & 9 \\
\hline 19 & T158M & 9 & 18 & 12 \\
\hline 20 & R270X & 7 & 12 & 10 \\
\hline 21 & $\mathrm{R} 255 \mathrm{X}$ & 8 & 12 & 6 \\
\hline 22 & R168X & 2 & 6 & 7 \\
\hline 23 & Deletion & 8 & 30 & 7 \\
\hline 24 & P405L & 6 & 18 & 5 \\
\hline 25 & Deletion & 11 & 33 & 8 \\
\hline 26 & R168X & 5 & 10 & 7 \\
\hline 27 & P322S & 12 & 15 & 5 \\
\hline 28 & R168X & 10 & 36 & 10 \\
\hline 29 & R294X & 4 & 14 & 8 \\
\hline 30 & P152A & 6 & 12 & 6 \\
\hline 31 & P152R & 11 & 12 & 11 \\
\hline
\end{tabular}

Note. RSSS refers to the summary score of the expanded Rett Syndrome Severity Scale (Kaufmann et al., 2012). 


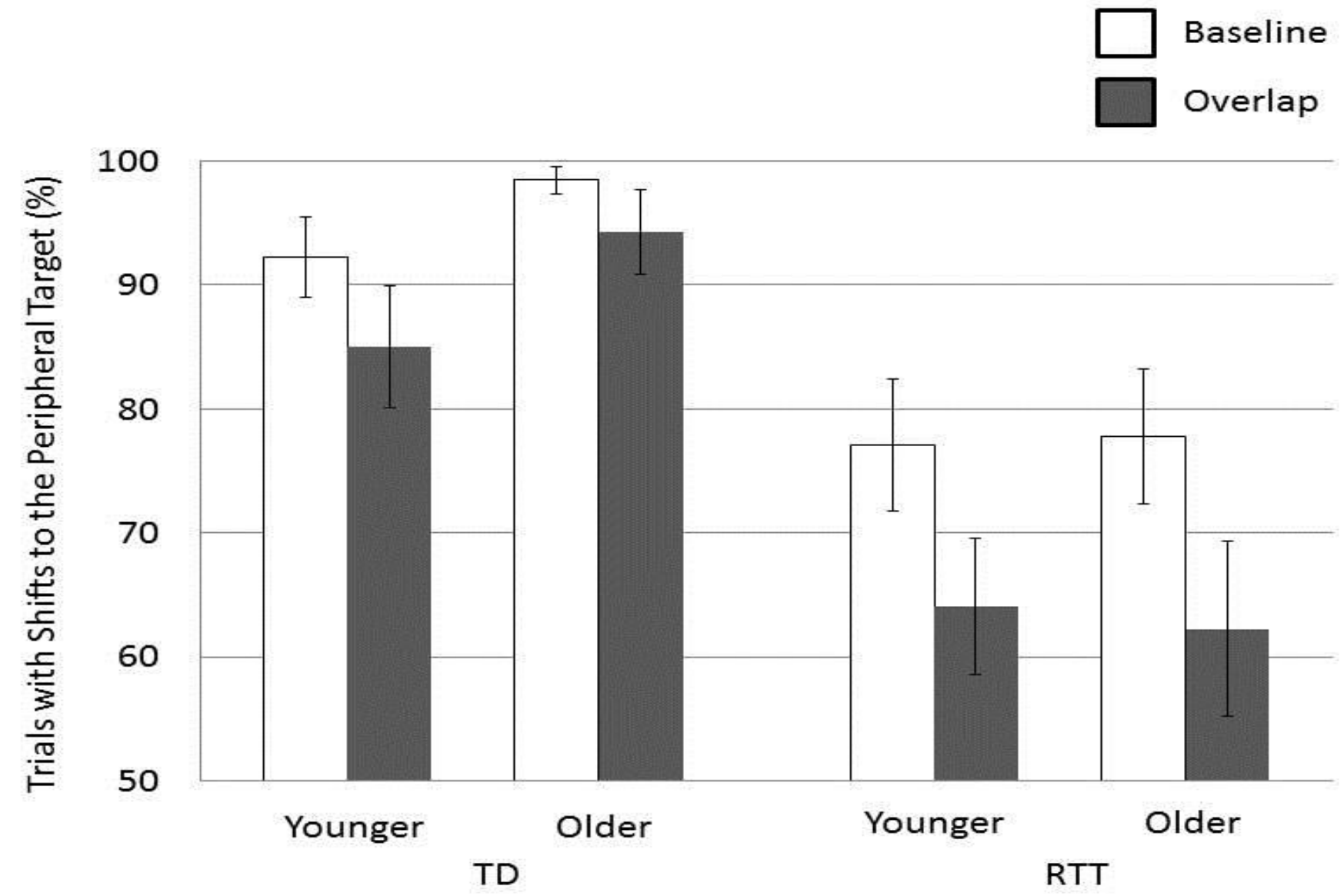

Fig 1 Frequency of gaze shifts on baseline and overlap trials for typically developing children (TD) and those with Rett Syndrome (RTT). 


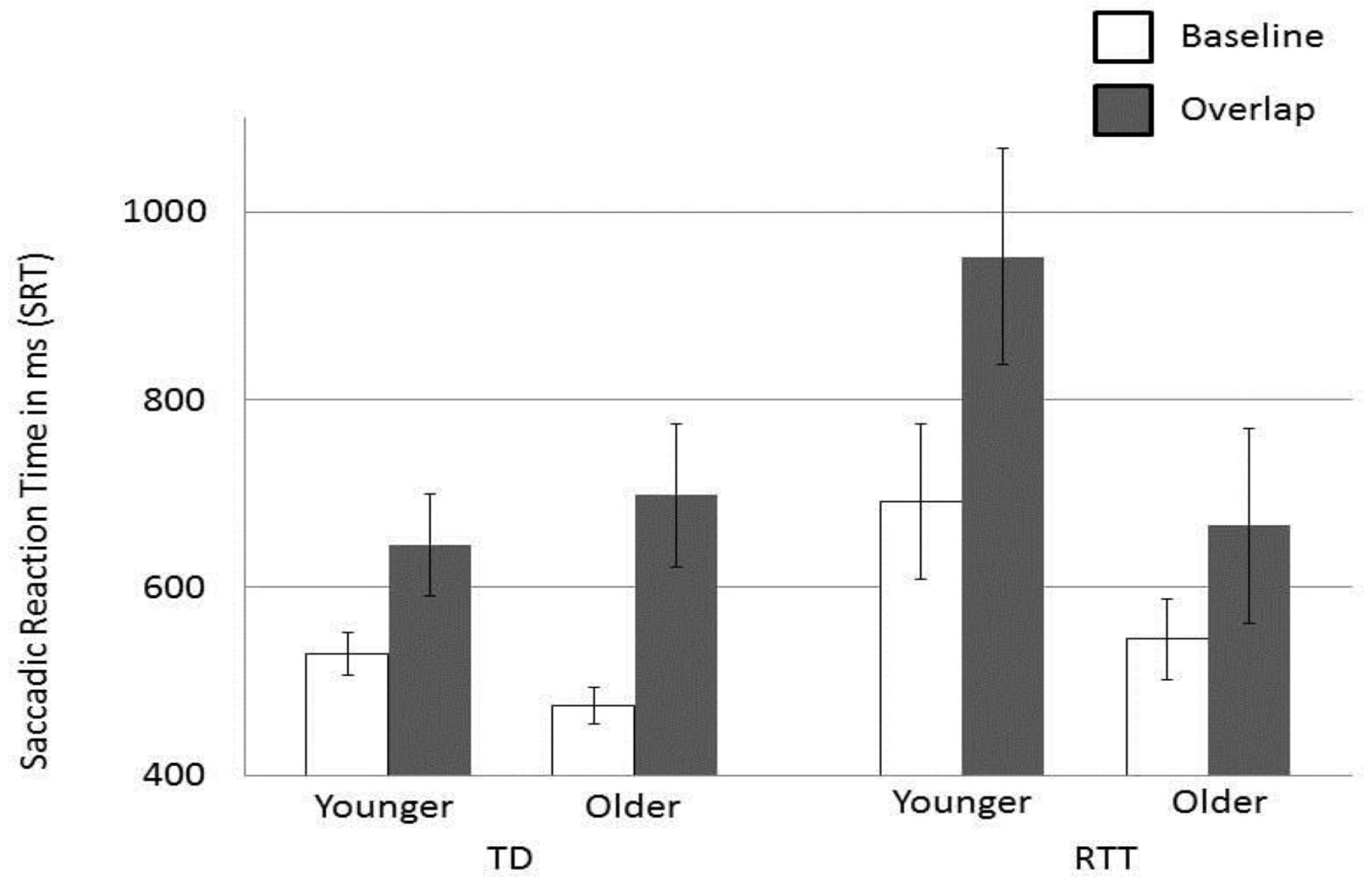

Fig 2: Saccadic reaction times on baseline and overlap trials for typically developing children (TD) and those with Rett Syndrome (RTT). 